\title{
Activation of mating type genes by transposition in Saccharomyces cerevisiae
}

\author{
(yeast/cell type/mating type switching/cryptic genes)
}

\author{
AMAR J. S. KLAR* AND SEYMOUR FOGEL ${ }^{\dagger}$ \\ *Cold Spring Harbor Laboratory, P. O. Box 100, Cold Spring Harbor, New York 11724; and †'Department of Genetics, University of California, \\ Berkeley, California 94720
}

Communicated by J. D. Watson, May 29, 1979

\begin{abstract}
Yeast Saccharomyces cerevisiae may express an a or $\alpha$ mating type. These cell types may be interconverted as a consequence of heritable genetic alterations at the mating type locus $(M A T)$. According to the more general controlling element model [Oshima, Y. \& Takano, I. (1971) Genetics 67, 327-335] and the specific cassette model [Hicks, J., Strathern, J. \& Herskowitz, I. (1977) in DNA Insertion Elements, Plasmids and Episomes, eds. Bukhari, A. I., Shapiro, J. A. \& Adhya, S. L. (Cold Spring Harbor Laboratory, Cold Spring Harbor, NY), pp. 457-462], the regulatory information required for switching the $M A T$ locus exists at two other loosely linked loci, $H M a$ and $H M \alpha$. Specifically, the $H M a$ and $H M \alpha$ loci are proposed to carry silent $\alpha$ and silent a genes, respectively. According to these models, switching occurs when a replica of a silent gene replaces the resident information at the mating type locus and is thereby expressed. These models predict that mutations at the silent ("storage") loci would generate defective MAT loci subsequent to the switching process. Therefore, the behavior of $H M \alpha$ mutants during the mating type interconversion was investigated. The results demonstrate that defective MATa alleles are generated by switching the $M A T \alpha$ locus in $H M \alpha$ mutants. Thus, the genetic information from $H M \alpha$ is transposed to the mating type locus. These results provide genetic evidence in support of these models.
\end{abstract}

Bakers yeast (Saccharomyces cerevisiae) may display either a or $\alpha$ mating types which are controlled by two allelic forms of the mating type locus (MAT), MAT a and MAT $\alpha$, respectively. During ordinary mitotic division cycles, heterothallic (ho) strains switch their mating type with a frequency of about $10^{-6}(1,2)$. In contrast, homothallic $(\mathrm{HO})$ strains may change frequently-as often as every cell generation (3-8). The mitotic products of a single haploid $H O$ cell may express opposite mating types and consequently fuse to produce MAT a/MAT $\alpha$ diploids. The MATa/MAT $\alpha$ diploids do not exhibit further switching. These switches comprise heritable stable changes at $M A T$, and the continued presence of the $\mathrm{HO}$ gene is not required for the maintenance of the altered allele $(5,6)$.

The MAT interconversion is promoted by genes $H O, H M \alpha$ (alternate allele $h m \alpha$ ), and $H M a$ (alternate allele $h m a$ ). The loci $H O$ and $H M \alpha$ or $h m a$ are required for switching $M A T \alpha$ to MAT a and $H O$ and $H M a$ or $h m \alpha$ are required for switching $M A T$ a to MAT $\alpha$ (9-12). The HM $\alpha$ and HMa loci map on opposite arms of chromosome III. To date, $\mathrm{HO}$ has not been mapped. MAT is situated about 20 map units from the centromere on the right arm of chromosome III $(11,12)$. Most heterothallic laboratory strains carry the genotype $H M a H M \alpha$ ho (Hawthorne, quoted in refs. 7 and 12).

Several models for mating type interconversion have been proposed (reviewed in refs. 7 and 13). These involve flip-flop controls, and it is proposed that both MAT alleles reside at MAT

The publication costs of this article were defrayed in part by page charge payment. This article must therefore be hereby marked "advertisement" in accordance with 18 U. S. C. $\$ 1734$ solely to indicate this fact. and they share a common regulatory site-e.g., promoter. In these models it is postulated that the switch is brought about by inverting the regulatory site by recombination or by DNA modification events $(7,14,15)$. Oshima and Takano (3) proposed the alternative controlling element model. They suggested that the $H M a$ and $H M \alpha$ loci code for mating type specific regulatory information. The attachment of the $H M a$ "controlling element" to MAT supposedly gives rise to the $M A T \alpha$ allele and the attachment of $H M \alpha$ controlling element forms the MAT a allele. $H O$ is suggested to control the insertion and removal of these elements at MAT.

Recently, Hicks et al. (4) proposed a more detailed version of the controlling element scheme. In their cassette model, $H M a$ and $h m \alpha$ are postulated to be blocks of unexpressed MAT $\alpha$ information and the $H M \alpha$ and $h m a$ loci are blocks of unexpressed MAT a information. It is suggested that the switch is brought about by insertion of this silent information or its copy (i.e., "cassette") into MAT with concomitant removal of the previous resident information. In effect, the activation of the silent information resident within the $H M a$ and $H M \alpha$ loci is promoted by the transpositions of their copies to the site of the mating type locus. A similar copying and transposition mechanism accounting for the genetic instabilities at the mating type locus in fission yeast was proposed by Egel (16).

Previously, we provided evidence consistent with the suggestion that the loci $H M \alpha$ and $h m a$ carry unexpressed MAT a information and that $H M a$ and $h m \alpha$ carry unexpressed $M A T \alpha$ information (17). The interpretation of the results of J. Rine, J. Strathern, J. Hicks, and I. Herskowitz (personal communication) is consistent with the suggestion that the cryptic $\alpha$ information exists at $H M a$. Subsequent to our studies and those of Rine et al., Haber and George (18) also suggested the existence of cryptic mating type information. A clear prediction of the more general controlling element model and the specific cassette model is that strains with mutations at the "storage" loci $H M a$ and $H M \alpha$ should generate defective MAT alleles after switching. In this communication we provide evidence that only defective MAT a alleles are obtained as a result of switching strains that are defective at the $H M \alpha$ locus.

\section{MATERIALS AND METHODS}

Strains. All strains used were Saccharomyces cerevisiae and are described in Table 1.

Construction of $H M \alpha$ Mutant Strains. Analysis of the proposed silent mating type loci at $H M a$ and $H M \alpha$ is made difficult by their cryptic nature. Recently, we found a mutation, marl (mating type regulator), that permits expression of the normally silent loci (17). We have used the marl mutant for isolating mutations of $H M a$ and $H M \alpha$ loci. Two independently isolated spontaneous mutations of $H M \alpha$, designated $H M \alpha^{\prime}-1$ and $H M \alpha^{\prime}-2$, were used in the present study. These mutations were transferred to strains containing the $\mathrm{HO}$ allele by standard genetic techniques. 
Table 1. List of strains used

\begin{tabular}{|c|c|c|}
\hline Strain & Genotype* & Source \\
\hline K62 & $H M a / H M a, M A T \mathbf{a} / M A T \alpha, H M \alpha^{\prime}-1 / H M \alpha^{\prime}-1, H O / H O$, cry1/cry1, thr4/thr4, ade6/ade6, his $2 /$ his 2, ura1/ura 1 & This study \\
\hline K63 & $H M a / H M a, M A T \mathbf{a} / M A T \alpha, H M \alpha^{\prime}-2 / H M \alpha^{\prime}-2, H O / H O$, leu2-1/leu2-1, tyr $7 /$ tyr 7 & This study \\
\hline $\mathrm{K} 40$ & $H M a, M A T \mathbf{a}, H M \alpha$, ho, aro1, his2 & This study \\
\hline KM2B-36D & $H M a, M A T \alpha, H M \alpha, h o, \operatorname{trp} 1$, ade6, ura1 & This study \\
\hline JC7 & $H M a, M A T \mathbf{a}, H M \alpha$, ho, kar1, leu1 & G. Fink \\
\hline K64 & $H M a$, mat $^{-}, H M \alpha$ or $H M \alpha^{\prime}-1$, ho, cry1, ura 1, his 2, ade 6 & This study \\
\hline K65 & $H M a$, mat $^{-}, H M \alpha$ or $H M \alpha^{\prime}-1$, ho, cry1, ura1, his2, aro1, thr4 & This study \\
\hline K66 & $H M a / H M a, M A T a / M A T \alpha, H M \alpha^{\prime}-1 / H M \alpha^{\prime}-1, H O / H O$, cry1/cry1, thr $4 /$ thr 4, ade6/ade6, his $2 / h i s 2$, ura $1 /$ ura 1 & This study \\
\hline
\end{tabular}

* The genetic symbols are those proposed by the Nomenclature Committee for Yeast Genetics (19) except the old terminology for the mating types and homothallism genes is retained (9).

Techniques. All media for growth and sporulation and techniques for micromanipulation and tetrad analysis have been described (20). Diploids were generated by cell-to-cell or cellto-spore matings as detailed (12). To test strains for the response to exogenous $\alpha$ factor, we arranged individual cells about $3 \mathrm{~mm}$ away from a streak of $\alpha$ cells that had grown overnight on rich medium. Production of $\alpha$ factor by the cells in the streak causes growth arrest of the a cells and induces a characteristic "shmoo" figure in less than $\approx 3$ hr of incubation (21). The growth of $\alpha$ cells and MAT a $/ M A T \alpha$ diploids is not arrested in the presence of the same $\alpha$ factor, nor do they exhibit the characteristic morphological alteration. Sensitivity to cryptopleurine was tested on media as described (22).

\section{RESULTS}

HMa, MAT $\alpha, H M \alpha^{\prime}$, HO Strains Yield Dual Mater Clones. Strains K62 and K66 (HMa/HMa, MAT a/MAT $\alpha$, $H M \alpha^{\prime}-1 / H M \alpha^{\prime}-1, H O / H O$; see Table 1 for complete genotype) and K63 (HMa/HMa, MATa/MAT $\alpha, H M \alpha^{\prime}-2 /$ $\left.H M \alpha^{\prime}-2, H O / H O\right)$ contain mutations at the $H M \alpha$ loci. Strains $\mathrm{K} 62, \mathrm{~K} 66$, and $\mathrm{K} 63$ were sporulated, dissected, and subjected to tetrad analysis. All of these strains yielded 2 MAT a/MAT $\alpha: 2$ "dual mater" segregant clones in each of 15 tetrads analyzed. The MAT a/MAT $\alpha$ segregant clones are non-mater and capable of sporulation. The dual mater clones mate efficiently with a and $\alpha$ tester strains and are incapable of sporulation. The subclones isolated from dual mater segregants continue to express this mating phenotype.

Homothallic diploid strains yield $2 \mathrm{a}: 2 \alpha$ spores. Generally, half of the spore progenies switch in the early generations after spore germination; consequently, cells of opposite mating type fuse to establish diploid MAT a/MAT $\alpha$ clones. We determined whether dual mater segregants arose exclusively from spores that were initially a or $\alpha$ or from both kinds of spores in strains with $H M \alpha^{\prime}$ mutations. Asci from strains K62 and K63 were dissected in the presence of $\alpha$ factor to assess the mating type of the spores. The $\alpha$ factor arrests the growth of the a spores whereas the $\alpha$ spores are unaffected (21). With this method, the mating type of the spores can be determined in about $4 \mathrm{hr}$. After determination of their mating type, the spores were grown in the absence of $\alpha$ factor. It was observed that the a spores produce non-mater clones which are capable of sporulation and the $\alpha$ spores yielded dual mater sporulation-negative progenies in 15 tetrads analyzed for each of strains K62 and K63. Therefore, we conclude that strains with genotype $H M a, M A T \alpha$, $H M \alpha^{\prime}, H O$ yield dual mater clones that are incapable of sporulation.

Strains K62 and K63 produced two segregants that grew at customary rates to establish colonies in about 3 days; the other two yielded much smaller colonies (Fig. 1). Without exception we found that the small colony size was associated with the dual mater clones. But, the non-mater sporulation-positive segregants grew to yield normal-size colonies. Closer examination of the dual mater segregants established that these clones contained both "a" and $\alpha$ cells. As a result, zygotic figures were abundantly present. The subclones isolated from these segregants continued to maintain the dual mating behavior. To account for this observation we suggest that the MAT a alleles obtained by switching $M A T \alpha$ in $H M \alpha^{\prime}$ mutants are defective. This supposition is readily accommodated within the framework of the cassette and controlling element models as follows.

The Indiscriminate Dual Mating Behavior of the HMa, $M A T \alpha, H M \alpha^{\prime}, H O$ Strains May Be Attributed to the Defective MATa Alleles Obtained by Switching. We may write the genotype of $H M a, M A T \alpha, H M \alpha^{\prime}, H O$ segregants in cassette terminology as $[\alpha] \alpha\left[\mathbf{a}^{-}\right] H O$. The [ ] allele signifies silent MAT information at $H M a([\alpha])$ and $H M \alpha([\mathbf{a}])$. The $\left[\mathbf{a}^{-}\right]$sig-

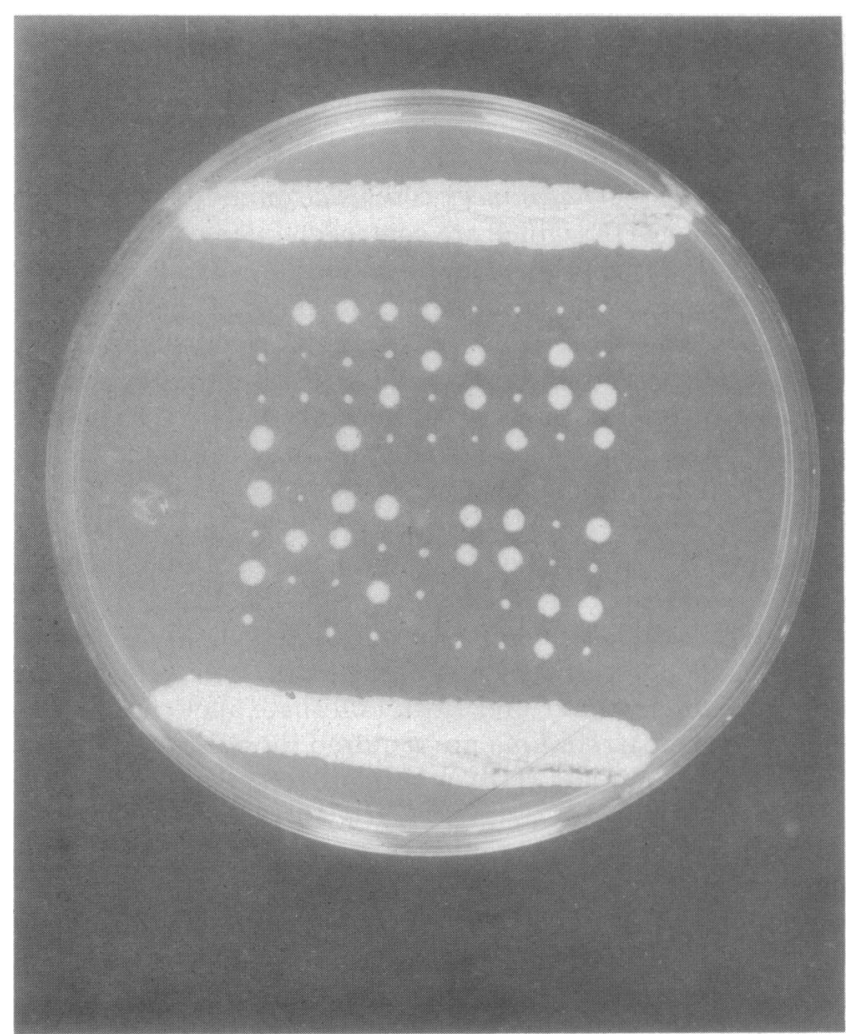

FIG. 1. Strain K63 (HMa/HMa, MATa/MAT $\alpha, H M \alpha^{\prime}-2 /$ $H M \alpha^{\prime}-2, H O / H O$ ) ascus dissection. Four spores from each ascus were planted on a horizontal line by micromanipulation. The picture was taken after 4 days of growth. Notice that each tetrad yielded two standard-size MAT $\mathbf{a} / M A T \alpha$ and 2 small dual mater segregant clones. The standard-size clones originated from the MAT a spores and the smaller segregant clones resulted from the growth of $M A T \alpha$ spores. 
nifies a mutation in the proposed silent MAT a information localized at $H M \alpha^{\prime}$. We postulate that the MAT $\alpha$ allele at the mating type locus can be switched to a mutant allele of MAT a $\left(\right.$ mat $\left.\mathbf{a}^{-}\right)$, the information for which is derived from the defective $H M \alpha$ locus. The cells with the mat $\mathrm{a}^{-}$allele can mate with their sister $\alpha$ cells to generate MAT $\alpha /$ mat $^{-}{ }^{-}$diploids (Fig. 2). Similar defective mat $\mathrm{a}^{-}$strains exhibiting an a mating phenotype have been described $(13,23)$. The MAT $\alpha /$ mat $^{-}$ cells exhibit an $\alpha$ phenotype $(13,23)$. With respect to recognition by the $H O$ system, these cells are probably indistinguishable from ordinary $\alpha$ cells and hence may switch again. Standard MAT a/MAT $\alpha$ cells do not exhibit switching. However, we and others have reported that diploid cells of the general type $M A T \alpha /$ mat $^{-}$can switch readily in the presence of the HO gene $(13,24)$. It would appear that mutations of the mat $\mathbf{a}^{-}$ type have lost the capacity to turn off switching in MAT $\alpha /$ mat $\mathbf{a}^{-}$diploids. The MAT $\alpha /$ mat $^{-}$cells can switch to mat $\mathrm{a}^{-} /$mat $^{-}$form because the same mutant $H M \alpha^{\prime}$ allele is used for obtaining mat $\mathrm{a}^{-}$information. The mat $\mathbf{a}^{-} /$mat $^{-} \mathbf{a}^{-}$ cells can switch back to MAT $\alpha /$ mat $^{-}$or to MAT $\alpha / M A T \alpha$ by switching one or both alleles of MAT to MAT $\alpha$. Also, these "a" and " $\alpha$ " cells can mate to yield tetraploids which are again available for switching. Consequently, we suggest that cells with genotype $H M a, M A T \alpha, H M \alpha^{\prime}, H O$ would grow, switch, mate, grow, switch, mate in a continuous process. The continuous switching and mating cycle is diagrammed in Fig. 2.

At any given time and as a result of cyclic mating and switching, the clone would have cells of both " $\alpha$ " and " $a$ " mating type-i.e., exhibit dual mating phenotype. Consequently, the progenies would mate continuously. Inevitably, the clones should show abundant zygotes and the size of the cells would increase due to the increase in ploidy. The predicted results were obtained. A lower growth rate of the dual mater segregant clones may be accounted for because they spend a considerable portion of their time in repeated switching and mating cycles.

These results are consistent with, but do not establish, that the defective mat $\mathrm{a}^{-}$alleles obtained by switching strains with $H M \alpha^{\prime}$ mutations reside at the mating type locus. Provided below is the evidence that establishes unequivocally that the defective mat $\mathbf{a}^{-}$alleles mapping at the mating type locus are obtained.

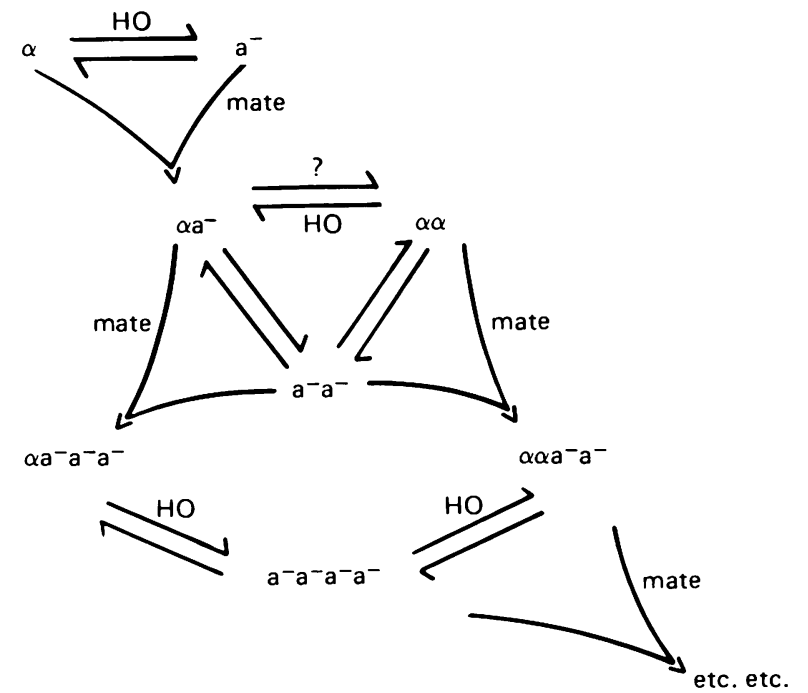

FI(i. 2. Indiscriminate dual mater yeast: a continuous switching/mating/growing cycle exhibited by $H M a, M A T \alpha, H M \alpha^{\prime}([\alpha][\alpha]$ $\left.\left|\mathbf{a}^{-}\right|\right) H()$ strains-an explanation for the phenotype. See text for details. The symbols $\alpha$ and $\mathbf{a}^{-}$represent MAT $\alpha$ and mat $\mathbf{a}^{-}$alleles, respectively.
Recovery of the Mutant mata ${ }^{-}$Allele and Its Mapping to the Mating Type Locus. The $\alpha$ spores obtained from strain K62 (HMa/HMa, cryl/cryl, MAT a/MAT $\alpha, H M \alpha^{\prime}-1 / H M \alpha^{\prime}-1$, $\mathrm{HO} / \mathrm{HO}$ ) were allowed to grow and switch, and the resulting zygotes obtained from matings between progenies of opposite mating type were placed adjacent to cells from a heterothallic strain K40 (HMa, CRY1, MAT a, HM $\alpha$, ho). If the zygotes were mostly MAT $\alpha /$ mat $^{-}$cells, they should express an $\alpha$ phenotype. The matings between zygotes or their immediate progeny with $\mathrm{K} 40$ ( $H M a, M A T$ a $, H M \alpha, h o)$ could be obtained easily by selecting for a complementation response of the various auxotrophic markers. Because the triploids MAT $\alpha /$ mat $^{-}-/$ MAT a $\mathrm{HO} / \mathrm{HO} /$ ho are not expected to exhibit switching, the mat $\mathrm{a}^{-}$ho segregants can be derived by ascus dissection and tetrad analysis. Three such triploid hybrids were constructed, sporulated, and dissected to assess the presence of mat $\mathbf{a}^{-}$allele. The hybrids yielded low spore viability (about $15 \%$ ), a result typical of triploids (20). A total of 144 meiotic segregants were tested for mating type; 104 segregants were observed to be either non-maters or dual maters, 12 had an $\alpha$ phenotype, and 22 had an a phenotype. The a segregants were tested for mat $\mathrm{a}^{-}$ phenotype. They were judged to carry an altered mat $\mathrm{a}^{-}$allele if, after mating with $\alpha$ cells, the resulting hybrids exhibited $\alpha$ mating type. In total, six segregants exhibited this property. Furthermore, all the mat $\mathrm{a}^{-}$segregants were resistant to cryptopleurine and, because strain $\mathrm{K} 62$ is cryl, we conclude that the mat $\mathrm{a}^{-}$allele derives from the $\mathrm{K} 62$ parent, the parent with the $H M \alpha^{\prime}-1$ mutation. cryl is a cryptopleurine-resistance marker about 2.5 centimorgans removed from MAT; segregation of this marker allows us to follow the closely linked MAT genotype of the spore segregants.

Hybrids between two such mat $\mathrm{a}^{-}$type segregants (strains K64 and K65, derived from two different triploids described above) and strain KM2B-36D (MAT $\alpha$ ho) were constructed by cell-to-cell mating. A technique was developed to sporulate

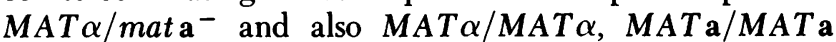
diploids which are ordinarily incapable of sporulation (unpublished data). The MAT $\alpha /$ mat $\mathbf{a}^{-}$cells were mated with JC7 (MAT a ho karl, courtesy of G. Fink). The karl mutant (karyogamy defective) strains can be conjugated with standard strains but the nuclei do not fuse (25). Consequently, one can construct heterokaryons with diploid MAT $\alpha /$ mat $^{-}{ }^{-}$and haploid MAT a, karl nuclei. The zygotes constructed by unions between MAT $\alpha /$ mat $^{-}{ }^{-}$and JC7 were directly inoculated to sporulation media. The diploid MAT $\alpha /$ mat $\mathbf{a}^{-}$nucleus can be induced to sporulate by virtue of the MAT a function provided by the MAT a, karl nucleus. The asci so obtained were dissected to map the mat $\mathrm{a}^{-}$allele unambiguously. Each of the hybrids between $\mathrm{K} 64$ and K65 with KM2B-36D yielded $2 \alpha: 2 \mathrm{a}$ segregants among 25 tetrads analyzed. All a segregants displayed the mat $\mathbf{a}^{-}$property - that is, after mating with $\alpha$ cells, the hybrids exhibited $\alpha$ mating type and were incapable of sporulation.

Furthermore, for the pairwise combination of mat $\mathrm{a}^{-}$and the cryl marker we obtained the following segregation data -24 parental ditype: 0 nonparental ditype: 2 tetratype tetrads for the K64 $\times$ KM2B-36D cross; and 25 parental ditype: 0 nonparental ditype: 2 tetratype for the K65 $\times$ KM2B-36D cross. Based on Perkins' (26) formula we calculate that mat $\mathbf{a}^{-}$ and cryl are closely linked and separated by about 3.8 centimorgans in both hybrids. A similar linkage of MAT to cryl has been reported (22). Because the defective mat $\mathrm{a}^{-}$mutation is allelic to $M A T \alpha$ and exhibits a rather close linkage to the $c r y l$ locus, we conclude that the switching of MAT $\alpha$ alleles in a $H M \alpha^{\prime}-1$ mutant yields defective mat $\mathbf{a}^{-}$alleles that map at MAT. 


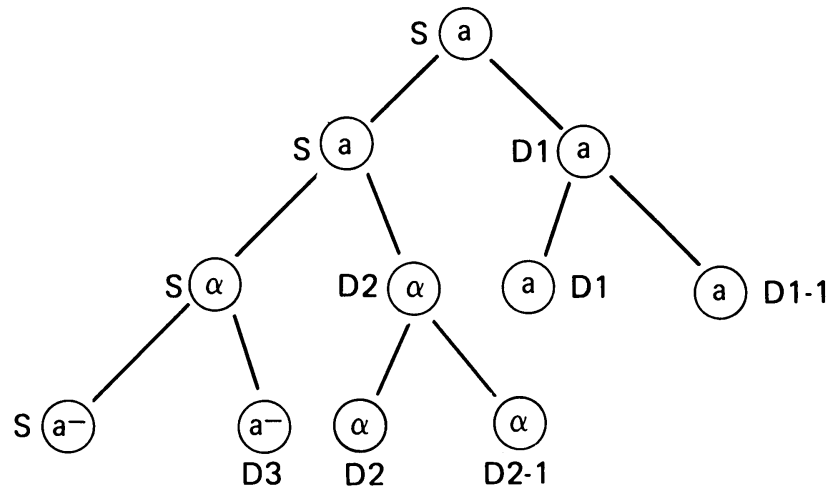

FIG. 3. Pedigree analysis of a spores derived from strain K66 $\left(H M a / H M a, M A T \mathbf{a} / M A T \alpha, H M \alpha^{\prime}-1 / H M \alpha^{\prime}-1, H O / H O\right)$. Progenies of the a spores were tested according to the procedure of Hicks and Herskowitz (7). S, spore cell; D1, first daughter; D2, second daughter; etc. D1-1, first daughter of D1. D1 and D1-1 grow to establish standard $M A T \mathrm{a} / M A T \alpha$ clones; $\mathrm{S}, \mathrm{D} 3, \mathrm{D} 2$, and D2-1 at the eight-cell stage grow to yield only dual mater clones. $\alpha, \mathbf{a}$, and $\mathbf{a}^{-}$represent $M A T \alpha, M A T \mathbf{a}$, and mat $^{-}{ }^{-}$, respectively.

Pedigree Analysis of $\boldsymbol{H M} \boldsymbol{\alpha}^{\prime}-1$ Mutant. Because bakers yeast grows by budding, and only a cells are arrested in growth by the $\alpha$ factor, one can easily distinguish the mating types of mother and daughter cells. Hicks and Herskowitz (7) exploited these properties to determine the pattern of mating type switching in cell pedigrees. They observed that the first two cells obtained from a homothallic spore do not exhibit a change in their mating type. However, in the next generation at the four-cell stage, in more than $80 \%$ of the pedigrees tested, the spore and its second bud switch whereas the first daughter and its bud do not change.

We determined whether the switching pattern of the $H M \alpha^{\prime}-1$ mutant is similar to that of the wild-type strain. Strain $\mathrm{K} 66$ (HMa/HMa, MAT a/MAT $\alpha, H M \alpha^{\prime}-1 / H M \alpha^{\prime}-1, H O /$ $H O)$ was sporulated and dissected and the switching pattern of the a and the $\alpha$ spore progenies was investigated. At the four-cell stage, two cells from each of the eight a spore progenies tested were observed to switch to $\alpha$ (Fig. 3). In each instance, the spore cell and its second bud exhibited the change. The first daughter and its bud, both of which were a cells, grew to produce sporulatable non-mater clones. Apparently, the switches of MAT a to functional MAT $\alpha$ occur in the $H M \alpha^{\prime}-1$ mutant. Some of the $\alpha$ cells derived from the a spore progenies switched to $a$ at the next generation. However, all of these secondary a cells and the $\alpha$ cells grew to produce dual mater clones incapable of sporulation. Furthermore, among $11 \alpha$ spores tested, 7 switched half of their progenies to a at the four-cell stage and the remainder at the eight-cell stage. All the a cells derived from the $\alpha$ progenies carried the mat $\mathrm{a}^{-}$allele because they grew to produce dual mater clones that were incapable of sporulation. Hence, the $\alpha$ progenies from K66 switch their MAT allele primarily or only to the $\mathrm{mat}^{-}$mutant form.

\section{DISCUSSION}

A salient feature of biological development is the process of cell differentiation. With respect to mating, S. cerevisiae may exhibit three cell types-a, $\alpha$, and sterile-as defined by the behavior of MAT a/MAT $\alpha$ diploids. The phenotypes are controlled by the mating type locus and can be interchanged by genetic changes at MAT in a nonrandom fashion. Studies by Hicks and Herskowitz (7) and Strathern and Herskowitz (8) on the pattern of mating type switching in the cell lineage revealed several analogies between $M A T$ interconversion and clonal differentiation in higher organisms. Because clonal differen- tiation is an essential feature in the development of higher organisms, $\mathrm{HO}$-mediated changes at $M A T$ represent an important model system for differentiation.

The cassette model for mating týpe interconversion was prompted by the observation that defective MAT $\alpha$ alleles can be readily switched to functional MAT a and subsequently to functional MAT $\alpha$-i.e., mutation within the MAT $\alpha$ locus can be "healed" by switching (refs. 7 and 24 , and Hawthorne quoted in ref. 7). Subsequently, we and others showed that defective MAT a alleles can be switched to functional MAT $\alpha$, which in turn can be switched to functional MAT a $(13,24)$. These observations are inconsistent with the flip-flop models proposed for mating type interconversion. To explain the healing effect, Hicks et al. (4) argued that the cells must have silent copies of MAT located elsewhere in the genome to provide genetic information during switching. Specifically, they proposed that the $H M a$ and $H M \alpha$ carry the silent copies of $M A T$. Recently, we provided evidence consistent with this suggestion (17). A further prediction of the cassette and the controlling element models is that the $H M a$ and $H M \alpha$ mutants should produce defective $M A T$ alleles after switching. Testing of this prediction constitutes a "wounding" experiment, which is a converse of the "healing" experiment (7). In this paper we provide results that bear on this prediction. It is clear that MAT $\alpha$ switches to defective (wounded) $\mathrm{mat}^{-}$in $H M \alpha \mathrm{mu}-$ tants. The defective MAT a alleles map at MAT. Hence, it is reasonable to conclude that a $H M \alpha$ mutant produces defective controlling elements or cassettes.

Three other independently isolated mutants of $H M \alpha$ similarly yield defective MAT a loci (data not shown). This evidence demonstrates that the mutations of the "storage" loci generate defective mating type alleles by switching. It is not known whether the molecular change in the $H M \alpha$ mutants studied is located in the coding or in the regulatory sequence of $M A T$ a information. Our unpublished results suggest that strains with nonsense mutations at $H M \alpha$ yield defective MAT a alleles carrying the corresponding nonsense mutations. Recently, $\mathrm{P}$. Kushner, L. Blair, and I. Herskowitz (personal communication) observed that the HMa mutant yields defective MAT $\alpha$ alleles by the interconversion process. In this communication the cassette model was treated as a specific version of the controlling element model. These data and our previous results (17) provide a body of genetic evidence supporting these models.

The authors thank Mrs. Jean McIndoo for technical assistance and Dr. John Game for critically reading the manuscript. Louisa Dalessandro prepared the manuscript. This investigation was supported by U.S. Public Health Service Grant GM-17317 to S.F. and Grant GM25678-01 to A.J.S.K

1. Hawthorne, D. (1963) Genetics 48, 1727-1729.

2. Rabin, M. (1970) Dissertation (Univ. Washington, Seattle, WA).

3. Oshima, Y. \& Takano, I. (1971) Genetics 67, 327-335.

4. Hicks, J., Strathern, J. \& Herskowitz, I. (1977) in DNA Insertion Elements, Plasmids and Episomes, eds. Bukhari, A. I., Shapiro, J. A. \& Adhya, S. L. (Cold Spring Harbor Laboratory, Cold Spring Harbor, NY), pp. 457-462.

5. Winge, O. \& Roberts, C. (1949) C. R. Trav. Lab. Carlsberg Ser. Physiol. 24, 341-346.

6. Hawthorne, D. (1963) Proc. Int. Congr. Genet. 11th 1, 34-35 (abstr.).

7. Hicks, J. \& Herskowitz, I. (1976) Genetics 83, 245-258.

8. Strathern, J. \& Herskowitz, I. (1979) Cell, 17, 371-381.

9. Harashima, S., Nogi, Y. \& Oshima, Y. (1974) Genetics 77, 639-650.

10. Naumov, G. I. \& Tolstorukov, I. I. (1973) Genetika 9, 82-91

11. Harashima, S. \& Oshima, Y. (1976) Genetics 84, 437-451.

12. Klar, A. J. S. \& Fogel, S. (1977) Genetics 85, 407-416. 
13. Klar, A. J. S., Fogel, S. \& Radin, D. N. (1979) Genetics, in press.

14. Hawthorne, D., in Holiday, R. \& Pugh, J. E. (1975) Science 187, 226-232.

15. Brown, S. (1976) J. Genet. 62, 81-91.

16. Egel, R. (1976) Mol. Gen. Genet. 145, 281-286.

17. Klar, A. J. S., Fogel, S. \& MacLeod, K. (1979) Genetics, in press.

18. Haber, J. E. \& George, J. P. (1979) Genetics, in press.

19. Plischke, M. E., von Borstel, R. C., Mortimer, R. K. \& Cohn, W. E. (1976) in Handbook of Biochemistry and Molecular Biology, ed. Fasman, G. D. (CRC, West Palm Beach, FL), 3rd Ed., pp 765-832.
20. Mortimer, R. K. \& Hawthorne, D. (1969) in The Yeasts, eds. Rose A. H. \& Harrison, J. S. (Academic, New York), Vol. 1, pp. 385460 .

21. Duntze, W., MacKay, V. \& Manney, T. (1970) Science 168, 1472.

22. Grant, P., Sanchez, L. \& Jimenez, A. (1974) J. Bacteriol. 120, 1308-1314.

23. Kassir, Y. \& Simchen, G. (1976) Genetics 82, 187-206

24. Strathern, J., Blair, B. \& Herskowitz, I. (1979) Proc. Natl. Acad. Sci. USA 76, 3425-3429.

25. Condi, J. \& Fink, G. (1976) Proc. Natl. Acad. Sci. USA 73, 3651-3655.

26. Perkins, D. D. (1949) Genetics 34, 607-626. 